\title{
Probabilistic Breakdown Phenomenon at On-Ramp Bottlenecks in Three-Phase Traffic Theory
}

\author{
Boris S. Kerner \\ DaimlerChrysler AG, REI/VF, HPC: G021, 71059 Sindelfingen, Germany \\ and \\ Sergey L. Klenov \\ Moscow Institute of Physics and Technology, Department of Physics, 141700 \\ Dolgoprudny, Moscow Region, Russia
}

\begin{abstract}
A nucleation model for the breakdown phenomenon in an initial non-homogeneous free traffic flow that occurs at an on-ramp bottleneck is presented. This model is in the context of three-phase traffic theory. In this theory, the breakdown phenomenon is associated with a first-order phase transition from the "free flow" phase to the "synchronized flow" phase. In contrast with many other nucleation models for phase transitions in different system of statistical physics in which random precluster emergence from fluctuations in an initial homogeneous system foregoes subsequent cluster evolution towards a critical cluster (critical nuclei), random precluster occurrence in free flow at the bottleneck is not necessary for traffic breakdown. In the model, the breakdown phenomenon can also occur if there were no fluctuations in free flow. This is because there is a permanent and motionless non-homogeneity that can be considered a deterministic vehicle cluster localized in a neighborhood of the bottleneck. The presented nucleation model and a nucleation rate of traffic breakdown that follows from the model exhibit qualitatively different features in comparison with previous results. In the nucleation model, traffic breakdown nucleation occurs through a random increase in vehicle number within the deterministic vehicle cluster, if the amplitude of the resulting random vehicle cluster exceeds some critical amplitude. The mean time delay and the associated nucleation rate of traffic breakdown at the bottleneck are found and investigated. The nucleation rate of traffic breakdown as a function of the flow rates to the on-ramp and upstream of the bottleneck is studied. Boundaries for traffic breakdown in the diagram of congested patterns at the bottleneck are found. These boundaries are qualitatively correlated with numerical results of simulation of microscopic traffic flow models in the context of three-phase traffic theory.
\end{abstract}




\section{Introduction}

Empirical observations of freeway traffic made in various countries show that the onset of congestion in an initial free flow is associated with an abrupt decrease in vehicle speed. This traffic breakdown called the "breakdown phenomenon" occurs mostly at freeway bottlenecks, in particular on-ramp bottlenecks. The traffic breakdown is accompanied by a hysteresis effect (see references in the reviews $[1,2]$, the book [3], and the conference proceedings $[4,5,6]$ ). The breakdown phenomenon has a probabilistic nature $[7,8,9]$ : At the same on-ramp bottleneck, traffic breakdown is observed at different flow rates in different realizations (days). The probability of the breakdown is a strong increasing function of flow rate downstream of the bottleneck $[8,9]$.

Most microscopic, macroscopic, probabilistic, and other models of freeway traffic explain the onset of congestion in free flow by moving jam emergence (see, e.g. [10,11,12,13,14,15] and references in the reviews $[16,17,18,19]$, as well as the conference proceedings $[20,21,22,23,24])$. In particular, in models of an on-ramp bottleneck moving jam(s) occurs spontaneously in free flow at the bottleneck when the flow rate upstream of the bottleneck is high enough and the flow rate to the on-ramp increases gradually beginning from zero $[17,18,25,26,28,27]$. However, the fundamental model result that the onset of congestion in free flow on a homogeneous road and at freeway bottlenecks is associated with spontaneous moving jam emergence $[10,13,14,15,25,26,28,27,16,17,18,19]$ is in a serious conflict with empirical evidence $[29,30,3]$.

Consequently, in 1996-1999 Kerner introduced three-phase traffic theory (see [3] for a review). In this theory, there are three traffic phases: free flow, synchronized flow, and wide moving jams. In accordance with empirical investigations of phase transitions, in this theory moving jams do not emerge spontaneously in free flow. Rather than moving jam emergence, a phase transition from free flow to synchronized flow ( $\mathrm{F} \rightarrow \mathrm{S}$ transition for short) governs the onset of congestion in free flow $[29,30]$. A first-order $\mathrm{F} \rightarrow \mathrm{S}$ transition postulated in threephase traffic theory [29] discloses the nature of the breakdown phenomenon at freeway bottlenecks found in empirical observations $[1,2]$. In other words, the terms "F $\rightarrow \mathrm{S}$ transition", "breakdown phenomenon", "speed breakdown", and "traffic breakdown" are synonyms. The first microscopic models in the context of three-phase traffic theory are stochastic models [31,32]. These models exhibit phase transitions as well as all types of congested patterns found in empirical observations $[31,32,33,34,3]$. Recently, some new microscopic models based on three-phase traffic theory have been developed $[35,36,37]$, which can show some congested pattern features found earlier in $[31,32]$.

One of the important methods for a study of phase transitions in non-linear distributed multiple-particle systems is a probabilistic theory based on an 
analysis of a master equation (e.g., $[38,39,40]$ ). First probabilistic theories for traffic flow based on a master equation for a random vehicle cluster have been introduced by Mahnke et al. [13,14] and further developed by Kühne et al. [15,41] (see the recent review by Mahnke et al. [40]). As usual for other metastable systems of statistical physics, for an initial metastable traffic flow a well-known two-well potential nucleation problem arises from the master equation, which analysis is made based on general well-known methods of statistical physics $[38,39]$. One of the main results of this analysis is the nucleation rate for the critical vehicle cluster (critical nuclei) whose occurence leads to a phase transition within the initial metastable state of traffic flow. As usual for other metastable systems of statistical physics [38,39], in the metastable traffic flow the nucleation rate for a phase transition is an exponential function of a control parameter, flow rate (or vehicle density) for traffic flow $[13,14,15,41,40]$. Rather than these common well-known results, an interest for the field has a nucleation model for a metastable traffic flow. Both the model and associated dependencies of the nucleation rates for phase transitions on traffic variables and control parameters should be adequate with real traffic flow features.

In $[13,14,15]$, models for vehicle cluster nucleation in an initial spatially homogeneous traffic flow on a homogeneous circular road are introduced (see, however, Sect. 4.2). One of the basic assumptions of these models is that in an initial homogeneous free flow firstly random precluster should emerge from fluctuations. In other words, this precluster foregoes subsequent cluster evolution towards a critical cluster (critical nuclei) whose growth leads to a phase transition. The rate of precluster emergence $w_{+}(0)$ in traffic, in which initially no vehicle cluster exists, can be different from the attachment rate of cluster evolution, when a random cluster with $n \geq 1$ vehicles already exists at the $\operatorname{road}[13,14,15,40]$.

A first nucleation model based on the master equation for traffic breakdown at an on-ramp bottleneck, i.e., in an open traffic system has been suggested by Kühne et al. [41] and Mahnke et al. (Chap. 17 of Ref. [40]). As in the models of homogeneous road $[13,14,15]$, in this model at given flow rates to the on-ramp $q_{\text {on }}$ and on the main road upstream of the on-ramp $q_{\text {in }}$ random vehicle cluster occurrence and evolution that can lead to traffic breakdown are realized only after a vehicle precluster consisting of one vehicle randomly appears at the bottleneck. The rate of precluster emergence is equal to the flow rate to the on-ramp $[41,40]: w_{+}(0)=q_{\text {on }}$. This idea about foregoing precluster emergence is associated with a basic model assumption that at $q_{\text {on }}=$ 0 no vehicle cluster can randomly appear, specifically no traffic breakdown is possible $[41,40]$. Probably, this basic model assumption leads to the nucleation rate for traffic breakdown that is proportional to $q_{\text {on }}[41,40]$. However, in real traffic flow both flow rates $q_{\text {on }}$ and $q_{\text {in }}$ exhibit random fluctuations that can cause cluster emergence. 
Moreover, whereas the basic assumption about the necessity of precluster emergence is physically justified for a homogeneous road $[13,14,15]$, this is not the case for for traffic breakdown at the bottleneck. This is because in contrast with the model of a homogeneous road [13,14,15], an initial state of free flow at the bottleneck at $q_{\mathrm{on}}>0$ and $q_{\text {in }}>0$ is non-homogeneous regardless of fluctuations [3]. This means that even there were no fluctuations in free flow at the bottleneck, nevertheless free flow is non-homogeneous in a neighborhood of the bottleneck. This is because two different flows permanent merge within the merging region of the on-ramp - the flow with the rate $q_{\text {on }}$ and the flow with the rate $q_{\text {in }}$.

It has been shown in three-phase traffic theory [3] that due to the merging of these flows a steady state of free flow at the bottleneck is non-homogeneous: A permanent and motionless local perturbation (deterministic perturbation) occurs at the bottleneck. Within this perturbation that can be considered a deterministic vehicle cluster the speed is lower and density is greater than downstream of the cluster. This deterministic cluster can lead to traffic breakdown even if there were no random fluctuations at the bottleneck $[42,3]$. However, in the nucleation model $[41,40]$ the master equation is formulated for probability of a vehicle cluster, which can occur due to fluctuations only. We see that for an initially non-homogeneous traffic flow, which occurs at the bottleneck, another physical approach should be applied. This is due to at least two reasons mentioned above: (i) Regardless of fluctuations, there is already a vehicle cluster at the bottleneck that growth can lead to traffic breakdown: No vehicle precluster emerging from fluctuations is necessary for traffic breakdown. (ii) When influence of fluctuations on traffic breakdown is considered, fluctuations caused by both flow rates $q_{\text {on }}$ and $q_{\text {in }}$ should be taken into account.

Thus, in contrast with the nucleation model at the bottleneck of Ref. [41,40] a nucleation model that is adequate with empirical results for traffic breakdown at the bottleneck should consider vehicle cluster evolution in an initially non-homogeneous free traffic. This random vehicle cluster should include both the total vehicle number within an initial deterministic cluster and addition vehicles within the cluster, which occur due to fluctuations.

However, there are no nucleation models and associated probabilistic theories for an initially spatially non-homogeneous free flow. Moreover, structure non-homogeneities play also a very important role for phase transitions in many other multiple-particle systems of statistical physics like granular flow, biological physics, reaction-diffusion systems, etc. (see references, e.g. in $[20,21,22,23,24,43,44])$. Thus, it seems important to derive a probabilistic theory for traffic breakdown in non-homogeneous traffic flow.

In this paper, a nucleation model for the probabilistic breakdown phenomenon in an initially spatially non-homogeneous traffic flow at an on-ramp bottleneck 
is presented. The article is organized as follows. In Sect. 2, the nucleation model is considered. Nucleation rate and the mean time delay for traffic breakdown that result from this model are studied in Sect. 3. Results of the paper as well as their comparison with earlier nucleation models for traffic breakdown of Ref. $[13,14,15,41,40]$ are discussed in Sect. 4 .

\section{Nucleation model of traffic breakdown in initially non-homogeneous free flow at on-ramp bottleneck}

\subsection{Non-homogeniety in free flow at bottleneck as reason for vehicle cluster}

In accordance with three-phase traffic theory [42,3], in a nucleation model we assume that the breakdown phenomenon at an on-ramp bottleneck is associated with an initial non-homogeneity of free flow at the bottleneck. This non-homogeneity exists at the bottleneck even in a hypothetical case in which there were no fluctuations in traffic flow. In this hypothetical case, this nonhomogeneity can be considered a deterministic (permanent) local perturbation localized at the bottleneck. The non-homogeneity of an initial free flow at the bottleneck is caused by two flows, which merge at the bottleneck: (i) An on-ramp inflow with the rate $q_{\text {on }}$. (ii) A flow on the main road upstream of the bottleneck with the rate $q_{\text {in }}$. This flow merging occurs permanent and on the same freeway location (within an on-ramp merging region). For this reason, the non-homogeneity of free flow at the bottleneck is motionless and permanent (Fig. 1 (a)).

To explain features of this non-homogeneity, note that at a given high enough flow rate $q_{\text {in }}$ in free flow on the main road upstream of the bottleneck, vehicles that merge from the on-ramp onto the main road force the vehicles on the main road to decelerate in the vicinity of an on-ramp merging region. This deceleration leads to a local decrease in speed and consequently to a local increase in density in the vicinity of the bottleneck. As a result, a local perturbation, i.e., non-homogeneity in free flow appears.

If no fluctuations in free flow are considered, as mentioned above the nonhomogeneity in free flow in the form of a deterministic local perturbation occurs at the bottleneck. The speed $v_{\text {free }}^{(\mathrm{B})}$ and density $\rho_{\text {free }}^{(\mathrm{B})}$ within this deterministic perturbation correspond to the conditions

$$
v_{\text {free }}^{(\mathrm{B})}<v^{(\text {free })}, \rho_{\text {free }}^{(\mathrm{B})}>\rho^{(\text {free })},
$$

where $v^{\text {(free) }}$ and $\rho^{\text {(free) }}$ are the average vehicle speed and density in homogeneous free flow on the main road downstream of the perturbation (Fig. 1 

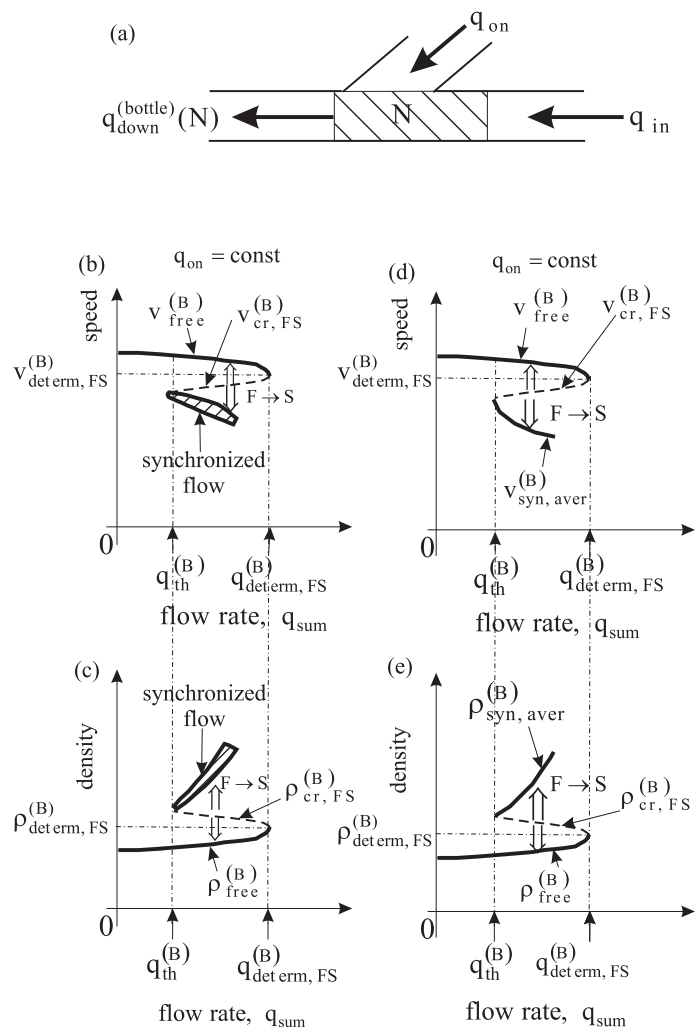

Fig. 1. Explanation of the basis of nucleation model: (a) Sketch of an on-ramp bottleneck. (b, c) - Z-shaped speed-flow (a) and the associated S-shaped density-flow characteristics for an $\mathrm{F} \rightarrow \mathrm{S}$ transition. (d, e) - Simplified Z-shaped speed-flow (d) and S-shaped density-flow characteristics (e) related to (b) and (c), respectively.

(a)). Because the deterministic local perturbation is permanent and motionless, at given $q_{\text {in }}$ and $q_{\text {on }}$, the total flow rate does not depend on the spatial co-ordinate, i.e., the speed and density on the main road satisfy the following condition:

$$
q_{\text {sum }}=v^{(\text {free })} \rho^{(\text {free })}=v_{\text {free }}^{(\mathrm{B})} \rho_{\text {free }}^{(\mathrm{B})},
$$

where

$$
q_{\mathrm{sum}}=q_{\mathrm{in}}+q_{\mathrm{on}}
$$

The inhomogeneous steady state within the deterministic local perturbation can be considered "deterministic vehicle cluster" in free flow localized at the bottleneck or "deterministic cluster" for short.

When $q_{\text {on }}$ is a given value and the total flow rate $q_{\text {sum }}$ increases, then the vehicle speed $v_{\text {free }}^{(\mathrm{B})}$ within the deterministic cluster decreases and in accordance with (2) the associated density $\rho_{\text {free }}^{(\mathrm{B})}$ increases. However, this increase is limited by 
some critical density $\rho_{\text {free }}^{(\mathrm{B})}=\rho_{\text {determ, FS }}^{(\mathrm{B})}$ (Fig. $\left.1(\mathrm{c})\right)$ within the deterministic cluster associated with a critical flow rate

$$
q_{\mathrm{sum}}=q_{\mathrm{determ}, \mathrm{FS}}^{(\mathrm{B})}
$$

After this critical deterministic perturbation is reached, the further increase in $q_{\text {sum }}$ leads to deterministic traffic breakdown at the bottleneck causing spontaneous synchronized flow emergence at the bottleneck. The critical deterministic perturbation can be considered "critical deterministic vehicle cluster" ("critical deterministic cluster" for short) or "deterministic nuclei for traffic breakdown". After the critical deterministic cluster is reached, deterministic traffic breakdown occurs at the bottleneck even if there were no random perturbations in traffic flow at the bottleneck.

Random perturbations within the initial deterministic cluster can cause random traffic breakdown $(\mathrm{F} \rightarrow \mathrm{S}$ transition) at the flow rate

$$
q_{\text {sum }}<q_{\text {determ, FS }}^{(\mathrm{B})}
$$

i.e., before the deterministic nuclei for traffic breakdown is reached. In this case, random traffic breakdown nucleation can occur at the bottleneck (arrows labeled $\mathrm{F} \rightarrow \mathrm{S}$ in Fig. 1 (b, c)). This is realized, if through a random increase in vehicle number within the initial deterministic cluster, the amplitude of the resulting "random vehicle cluster" ("random cluster" for short) exceeds some critical amplitude associated with a critical density within the random cluster

$\rho_{\mathrm{cr}, \mathrm{FS}}^{(\mathrm{B})}($ Fig. $1(\mathrm{c}))$. The random cluster with the critical density $\rho_{\mathrm{cr}, \mathrm{FS}}^{(\mathrm{B})}$ can be considered "critical random vehicle cluster" at the bottleneck ("critical random cluster" for short) or "random nuclei for traffic breakdown". If the amplitude of a random cluster is smaller than the critical one, the random cluster decays towards the initial deterministic cluster.

\subsection{Master equation}

\subsubsection{Basic assumptions for master equation}

There are the following basic assumptions for the nucleation model in an initial non-homogeneous free flow at the bottleneck:

(i) There is no vehicle precluster, which random occurrence through a random decrease in speed of one of the vehicles in the initial free flow should be necessary for vehicle cluster emergence and subsequent cluster evolution. Traffic breakdown occurs within a deterministic vehicle cluster that is motionless and permanently exists in a neighborhood of the bottleneck. 


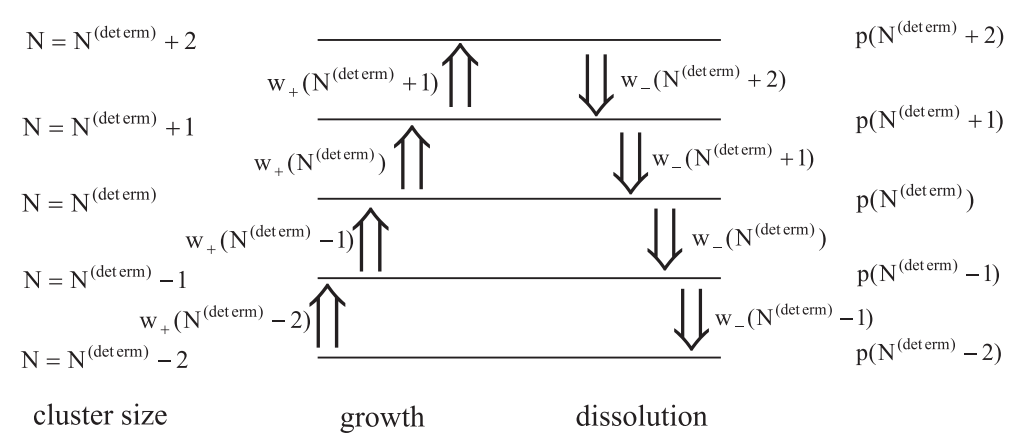

Fig. 2. Schematic illustration of cluster transformation in an initially non-homogeneous free flow at the bottlneck.

(ii) A master equation should describe probability $p$ for random vehicle cluster evolution in which the cluster size $N$, i.e., the total vehicle number within the motionless vehicle cluster randomly changes due to fluctuations in a neighborhood of the deterministic cluster (Fig. 2). The size of the deterministic cluster $N^{\text {(determ) }}$ does not depend on fluctuations in traffic flow.

(iii) The attachment rate $w_{+}$onto this vehicle cluster is not a function of the cluster size $N$, i.e. $w_{+}(0)=w_{+}(N)=q_{\text {in }}+q_{\text {on }}$.

(iv) A dependence of the detachment rate $w_{-}$from a cluster is a non-linear function on $N$, which consists of at least two branches associated with the deterministic cluster and a critical vehicle cluster (nuclei for traffic breakdown).

(v) The on-ramp inflow and the flow upstream of the bottleneck make a different influence on the cluster size. To take into account this assymetric behavior in the model, the detachment rate $w_{-}$also depends on $q_{\mathrm{on}}$.

The assumption (i) can be explained by existence of a deterministic vehicle cluster at the bottleneck. This cluster can lead to traffic breakdown at the bottleneck even there were no fluctuations in traffic flow, i.e., no precluster is necessary for vehicle cluster occurrence in an initially steady state of nonhomogeneous free flow at the bottleneck. To explain the assumption (ii), note that real random fluctuations can cause either a decrease or an increase in the cluster size $N$ and, respectively, either a decrease or an increase in vehicle density within the cluster. Real random fluctuations, which decrease the cluster size $N$, are associated with an increase in speed within the cluster (Sect. 2.1). Therefore, these fluctuations can prevent the deterministic breakdown. In contrast, random fluctuations, which increase the cluster size $N$, i.e., decrease the speed within the cluster, can cause traffic breakdown before the deterministic nuclei for traffic breakdown is reached, i.e., when the condition (5) is satisfied. Regardless of the cluster size $N$, the attachment rate into the cluster is determined by fluctuations in both flow rates $q_{\text {in }}$ and $q_{\text {on }}$. This explains the assumption (iii). Grounds for the assumptions (iv) and (v) appear in Sect. 2.2.2. 
We consider the dynamics of the total vehicle number $N$ within a vehicle cluster localized at the bottleneck (dashed region in Fig. 1 (a)). It is assumed that within the cluster free flow is spatially non-homogeneous, specifically the region of spatial cluster localization is bounded upstream and downstream with homogeneous free flows. The total number of vehicles $N$ within the cluster can either increase or decrease over time randomly in comparison with a value $N=N^{\text {(determ) }}$ for the case in which the deterministic cluster exists at the bottleneck only.

In accordance with the above basic assumptions, the probability $p(N, t)$ to find $N$ vehicles within the cluster at the bottleneck reads as follows

$$
\begin{array}{r}
\frac{\partial p(N, t)}{\partial t}=w_{+}(N-1) p(N-1, t)+w_{-}(N+1) p(N+1, t) \\
-\left[w_{+}(N)+w_{-}(N)\right] p(N, t), \quad \text { at } N>0, \\
\frac{\partial p(0, t)}{\partial t}=w_{-}(1) p(1, t)-w_{+}(0) p(0, t), \quad \text { at } N=0,
\end{array}
$$

with the boundary condition

$$
w_{-}(0)=0
$$

In accordance with the assumption (3), the vehicle attachment rate $w_{+}$is independent of $N$, i.e.,

$$
w_{+}=q_{\mathrm{sum}} .
$$

\subsubsection{Vehicle detachment rate from cluster}

The vehicle detachment rate $w_{-}$is obviously equal to the outflow rate from the cluster

$$
w_{-}(N)=q_{\text {down }}^{(\text {bottle })}(N)
$$

To understant a qualitative shape of the function $q_{\text {down }}^{\text {(bottle) }}(N)$, note that in accordance with three-phase traffic theory [3], speed states within the deterministic cluster $v_{\text {free }}^{(\mathrm{B})}\left(q_{\text {sum }}\right)$, the speed within the critical random cluster $v_{\text {cr, FS }}^{(\mathrm{B})}\left(q_{\text {sum }}\right)$, along with a 2D synchronized flow speed states [3], together form a Z-shaped speed-flow characteristic for traffic breakdown (Fig. 1 (b)). The associated density-flow characteristic, which consists of density states within 
the deterministic cluster $\rho_{\text {free }}^{(\mathrm{B})}\left(q_{\text {sum }}\right)$, the density within the critical random cluster $\rho_{\text {cr, FS }}^{(\mathrm{B})}\left(q_{\text {sum }}\right)$, along with a $2 \mathrm{D}$ synchronized flow states, has obviously a S-shaped form (Fig. 1 (c)) ${ }^{1}$ Due to an $\mathrm{F} \rightarrow \mathrm{S}$ transition, fast cluster growth at the bottleneck occurs leading to congested pattern formation, i.e., either a synchronized flow pattern (SP) or a general pattern (GP) appears upstream of the bottleneck [3]. However, the nucleation effect leading to traffic breakdown and its characteristics are fully independent of possible congested patterns resulting from this $\mathrm{F} \rightarrow \mathrm{S}$ transition. For this reason, we can average the infinity of synchronized flow states (dashed regions in $(b, c)$ ) for each given flow rate $q_{\text {sum }}$ to one speed $v_{\text {syn, aver }}^{(\mathrm{B})}\left(q_{\text {sum }}\right)$ and to one density $\rho_{\text {syn, aver }}^{(\mathrm{B})}\left(q_{\text {sum }}\right)($ Fig. $1(\mathrm{~d}$, e) ${ }^{2}$. A consideration of the resulting congested patterns is beyond the scope of this article.

In the model, it is assumed that the shape of the characteristic $q_{\text {down }}^{\text {(bottle) }}(N)$ (Fig. 3) follows from the S-shaped density-flow characteristic of three-phase traffic theory (Fig. $1(\mathrm{e})$ ): The characteristic $q_{\text {down }}^{\text {(bottle) }}(N)$ has at least two different branches $q_{\text {down }}^{\text {(bottle) }}(N)$ labeled $N^{\text {(determ) }}$ and $N_{\mathrm{c}}$ in Fig. 3 (a). These branches are related to the vehicle number ranges, respectively, given by the conditions

$$
0 \leq N \leq N_{\mathrm{d}}
$$

and

$$
N_{\mathrm{d}}<N \leq N_{\mathrm{s}} .
$$

The branches $N^{\text {(determ) }}$ and $N_{\text {c }}$ in Fig. 3 (a) are associated with the density branches $\rho_{\text {free }}^{(\mathrm{B})}$ and $\rho_{\text {cr, FS }}^{(\mathrm{B})}$ of the S-shaped density-flow characteristic in Fig. 1 (e), respectively. The branch $N^{(\text {determ) }}$ is associated with the case in which at a high enough flow rate $q_{\text {sum }}$ and the on-ramp flow rate $q_{\text {on }}>0$ the deterministic cluster exists at the bottleneck. The branch $N_{\mathrm{c}}$ is associated with the case in which the critical random cluster whose growth leads to an $\mathrm{F} \rightarrow \mathrm{S}$ transition occurs at the bottleneck.

1 Within the deterministic cluster the speed $v_{\text {free }}^{(\mathrm{B})}$ and density $\rho_{\text {free }}^{(\mathrm{B})}$ shown in Fig. 1 (b-e) are connected by the formula (2). The number of vehicles within the cluster $N=\int \rho d x$, where integration is performed over the region of cluster localization.

2 Concerning synchronized flow states in the vicinity of the bottleneck associated with a resulting congested pattern, we should note that assumptions used in our nucleation model are satisfied only for a localized SP (LSP) [3]. An LSP is an SP whose downstream front is fixed at the bottleneck. The upstream front of the LSP is localized on the main road at some finite distance upstream of the bottleneck, i.e., the width (in the longitudinal direction) of the LSP is always limited. Synchronized flow states at the bottleneck are drawn in Fig. 1 (b, c) and in other illustrations only for the case in which the congested pattern is an LSP. 

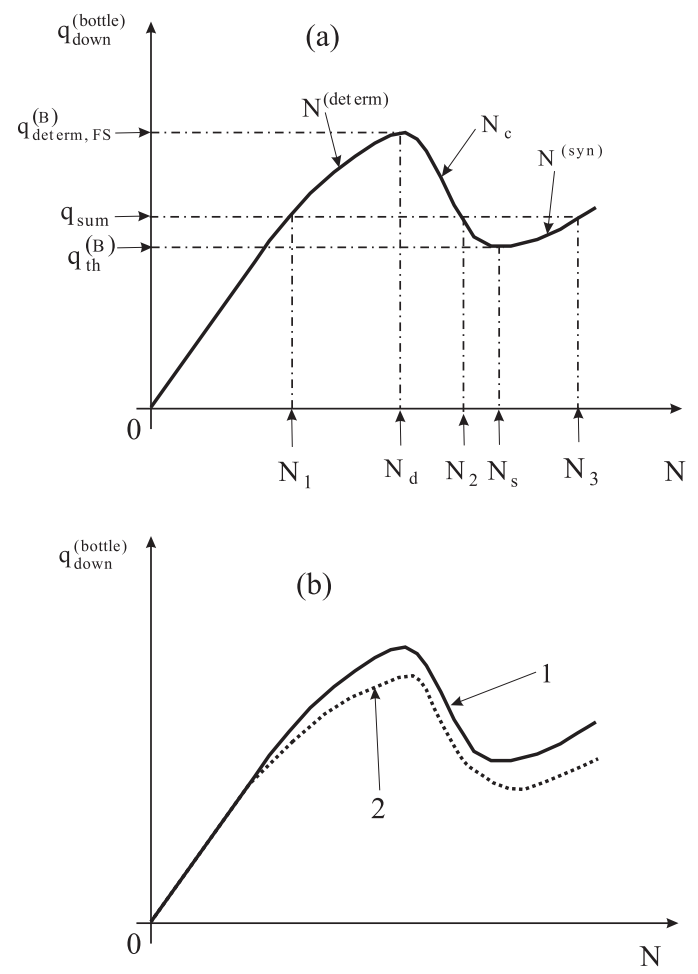

Fig. 3. Qualitative dependencies of the outflow rate $q_{\text {down }}^{\text {(bottle) }}$ on the total vehicle number $N$ within the cluster localized at the bottleneck (a), and possible dependencies of the N-shaped function $q_{\text {down }}^{\text {(bottle) }}(N)$ on $q_{\text {on }}$ for two different values $q_{\text {on }}(\mathrm{b})$; curve 1 for $q_{\mathrm{on}}=q_{\mathrm{on}}^{(1)}$, curve 2 for $q_{\mathrm{on}}=q_{\mathrm{on}}^{(2)}>q_{\mathrm{on}}^{(1)}$.

In addition, from the S-shaped density-flow characteristic (Fig. 1 (e)) can be seen that for the case in which an LSP results from an $\mathrm{F} \rightarrow \mathrm{S}$ transition, at

$$
N>N_{\mathrm{s}},
$$

there is a third branch $N^{(\text {syn) }}$ on the characteristic $q_{\text {down }}^{(\text {bottle) }}(N)$ (Fig. 3 (a)) associated with the branch $\rho_{\mathrm{syn}}^{(\mathrm{B})}$, aver for averaged synchronized flow states in Fig. 1 (e). In this case, $q_{\text {down }}^{\text {(bottle) }}(N)(10)$ is a N-shaped flow-vehicle-number characteristic.

At the critical point $N=N_{\mathrm{d}}$ at which the branches $N^{\text {(determ) }}$ and $N_{\mathrm{c}}$ merges, the function $q_{\text {down }}^{\text {(bottle) }}(N)$ has its maximum point. At the threshold point $N=N_{\mathrm{s}}$ at which the branches $N_{\mathrm{c}}$ and $N^{(\mathrm{syn})}$ merges, the function $q_{\text {down }}^{\text {(bottle }}(N)$ has its minimum point.

Quantitative characteristics of the N-shaped function $q_{\text {down }}^{\text {(bottle) }}(N)$ (e.g., values $N_{\mathrm{d}}$ and $N_{\mathrm{s}}$ ) can depend on the flow rate to the on-ramp $q_{\text {on }}$ (assumption (v) in Sect. 2.2.2). This is because the on-ramp inflow and the flow upstream of the bottleneck can make a considerable different influence on the cluster size and the outflow rate from the cluster $q_{\text {down }}^{\text {(bottle) }}$. In particlular, it can turn out 
that at the same $N$ the greater $q_{\text {on }}$, the more difficult for vehicles to escape from the cluster, i.e., the less $q_{\text {down }}^{\text {(bottle) }}$ is. This is confirmed by microscopic simulations [32] and reflected in Fig. 3 (b) in which it is assumed that the greater $q_{\text {on }}$, the greater $N_{\mathrm{d}}$ and $N_{\mathrm{s}}$ are. Thus, in a general case instead of (10) we should use

$$
w_{-}(N)=q_{\text {down }}^{(\text {bottle })}\left(N, q_{\text {on }}\right)
$$

A possible impact of the flow rate $q_{\text {on }}$ on quantitative characteristics of the mean time delay for an $\mathrm{F} \rightarrow \mathrm{S}$ transition is discussed in Sect. 4 .

Through the use of the basic assumptions (i)-(v) of Sect. 2.2.1 and the chosen shape of the function (10) discussed above (Fig. 3 (a)), critical cluster occurrence describes an $\mathrm{F} \rightarrow \mathrm{S}$ transition at the bottleneck. There are two reasons for this statement: (i) A vehicle cluster is motionless, i.e., fixed at the bottleneck. This is related to the definition of synchronized flow whose downstream front is usually fixed at the bottleneck, whereas the downstream from of a wide moving jam propagates trough any bottleneck while maintaining the mean downstream jam velocity [3]. (ii) The shape of the chosen function (10) in the nucleation model associated with this motionless cluster (Fig. 3 (a)) follows from a Z-shaped speed-flow characteristic for traffic breakdown (Fig. 1 (b)) for an $\mathrm{F} \rightarrow \mathrm{S}$ transition at the bottleneck found in a microscopic traffic flow theory. In this theory has been shown that if these two requirements are satisfied, then rather than an $\mathrm{F} \rightarrow \mathrm{J}$ transition (moving jam emergence) an $\mathrm{F} \rightarrow \mathrm{S}$ transition, i.e., synchronized flow emergence occurs at the bottleneck. As follows from this microscopic theory, after the $\mathrm{F} \rightarrow \mathrm{S}$ transition has already occurred, moving jams can emerge in this synchronized flow. However, the nucleation model describes traffic breakdown, i.e., an $\mathrm{F} \rightarrow \mathrm{S}$ transition, specifically the rate of traffic breakdown (synchronized flow) nucleation only.

In addition, it should be noted that the branch for synchronized flow $v_{\mathrm{syn}}^{(\mathrm{B})}$ aver in

Figs. 1 (b-e) and the associated branch $N^{\text {(syn) }}$ on the characteristic $q_{\text {down }}^{\text {(bottle) }}(N)$ (Fig. 3 (a)) follow from the microscopic traffic theory, rather than from the nucleation model. This branch, which is shown only with the aim of a qualitative illustration of a possible traffic flow state after synchronized flow nucleation, has no influence on the nucleation rate of an $\mathrm{F} \rightarrow \mathrm{S}$ transition.

\subsection{Steady states}

Steady states of vehicle number $N$ at given $q_{\text {in }}$ and $q_{\text {on }}$ are associated with solutions of the equation 


$$
w_{+}=w_{-}(N)
$$

In accordance with (9), (14), they are found from the condition

$$
q_{\text {sum }}=q_{\text {down }}^{(\text {bottle })}\left(N, q_{\text {on }}\right) .
$$

As can be seen from Fig. 3 (a), at given flow rates $q_{\text {on }}$ and $q_{\text {in }}$ that satisfy the condition

$$
q_{\mathrm{th}}^{(\mathrm{B})}<q_{\mathrm{sum}}<q_{\text {determ, FS }}^{(\mathrm{B})}
$$

there can be at least two steady states: $N=N_{1}$ associated with the deterministic cluster and $N=N_{2}$ associated with the critical random cluster. These steady states are the roots of Eq. (16), i.e., they are associated with the intersection points of the horizontal line $q_{\text {down }}^{\text {(bottle) }}=q_{\text {sum }}$ with the branches $N^{\text {(determ) }}$ and $N_{\text {c }}$ of the characteristic $q_{\text {down }}^{\text {(bottle) }}\left(N, q_{\text {on }}\right)$ (Fig. $3($ a) $)$, respectively. In addition, if an LSP occurs as a result of an $\mathrm{F} \rightarrow \mathrm{S}$ transition, then there is a third root of Eq. (16), $N=N_{3}$, associated with the intersection point of the horizontal line $q_{\text {down }}^{\text {(bottle) }}=q_{\text {sum }}$ with the branch $N^{(\text {syn) }}$ of the characteristic $q_{\text {down }}^{\text {(bottle) }}(N)$.

If the flow rate $q_{\text {sum }}$ increases, then the critical vehicle number difference within the cluster

$$
\Delta N_{\mathrm{c}}=N_{2}-N_{1}
$$

decreases. This critical vehicle number difference is associated with the vehicle number difference within the critical random cluster and within the initial deterministic cluster at the bottleneck. The growth of the critical random cluster leads to traffic breakdown at the bottleneck.

At the critical flow rate (4), we get $\Delta N_{\mathrm{c}}=0$ : The steady states $N_{1}$ and $N_{2}$ merge into one point with the critical vehicle number $N=N_{\mathrm{d}}$ at which $q_{\text {determ, FS }}^{(\mathrm{B})}=q_{\text {down }}^{(\text {bottle })}\left(N_{\mathrm{d}}, q_{\mathrm{on}}\right)$. At $q_{\text {sum }} \geq q_{\text {determ, FS }}^{(\mathrm{B})}$ the deterministic traffic breakdown should occur even if there is no random increase in the vehicle number within the initial deterministic cluster at the bottleneck.

If the flow rate $q_{\text {sum }}$ decreases gradually, then the threshold flow rate

$$
q_{\mathrm{sum}}=q_{\mathrm{th}}^{(\mathrm{B})}
$$

is reached at which the steady states $N_{2}$ and $N_{3}$ merge into one threshold steady state $N=N_{\mathrm{s}}$ at which $q_{\mathrm{th}}^{(\mathrm{B})}=q_{\text {down }}^{(\text {bottle })}\left(N_{\mathrm{s}}, q_{\mathrm{on}}\right)$. 

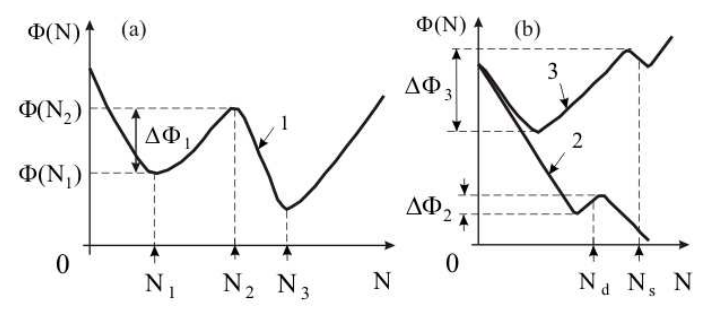

Fig. 4. Qualitative shape of the potential $\Phi(N)$ (22) for different flow rates $q_{\text {sum }}$ : curves 1,2 , and 3 are related to the corresponding flow rates $q_{\mathrm{sum}}^{(1)}, q_{\mathrm{sum}}^{(2)}$, and $q_{\mathrm{sum}}^{(3)}$ satisfying the condition $q_{\text {sum }}^{(3)}<q_{\text {sum }}^{(1)}<q_{\text {sum }}^{(2)}$.

\section{Nucleation rate of traffic breakdown at bottleneck}

As follows from the analysis of the model (6)-(9), (14) (Appendix A), in the flow rate range (17) the mean time delay of an $\mathrm{F} \rightarrow \mathrm{S}$ transition at the bottleneck is

$$
T_{\mathrm{FS}}^{(\mathrm{B}, \text { mean })}=C \exp \{\Delta \Phi\},
$$

where a potential barrier

$$
\Delta \Phi=\Phi\left(N_{2}\right)-\Phi\left(N_{1}\right)
$$

the potential $\Phi(N)$ is

$$
\begin{aligned}
& \Phi(N)= \begin{cases}\sum_{n=1}^{N} \ln \frac{w_{-}(n)}{w_{+}} \text {at } N>0, \\
0 & \text { at } N=0,\end{cases} \\
& C=2 \pi\left(w_{-}^{\prime}\left(N_{1}\right)\left|w_{-}^{\prime}\left(N_{2}\right)\right|\right)^{-\frac{1}{2}},
\end{aligned}
$$

$w_{-}^{\prime}(N)=d w_{-} / d N$. Respectively, the nucleation rate for traffic breakdown at the bottleneck is

$$
G_{\mathrm{FS}}^{(\mathrm{B})}=\frac{1}{T_{\mathrm{FS}}^{(\mathrm{B}, \text { mean })}}=C^{-1} \exp \{-\Delta \Phi\} .
$$

To find a qualitative shape $\Phi(N)(22)$ (Fig. 4), a change in $\Phi(N)$ between two neighboring points $N$ and $N-1$ that equals

$$
\delta \Phi(N)=\Phi(N)-\Phi(N-1)=\ln \frac{w_{-}(N)}{w_{+}}
$$


can be used. The value $\delta \Phi(N)(25)$ becomes zero at the maximum and minimum points of the function $\Phi(N)$, i.e., at the roots of Eq. (15) that are the points $N=N_{\mathrm{i}}, i=1,2,3$ discussed above (Fig. $3(\mathrm{a})$ ). The value $\delta \Phi(N)>0$ at $w_{-}(N)>w_{+}$, i.e., at points of the curve $w_{-}(N)$ above the horizontal line $q=q_{\text {sum }}$ in (Fig. $3\left(\right.$ a)). In contrast, $\delta \Phi(N)<0$ at $w_{-}(N)<w_{+}$, i.e., at points of the curve $w_{-}(N)$ below the horizontal line $q=q_{\text {sum }}$.

It can be seen from (20) that the mean time delay for traffic breakdown decreases exponentionally with increase in potential barrier $\Delta \Phi(21)$. If in Fig. 4 the total flow rate increases from $q_{\text {sum }}=q_{\text {sum }}^{(1)}$ to $q_{\text {sum }}=q_{\text {sum }}^{(2)}$, which is close to the critical flow rate (4) for deterministic traffic breakdown, then the potential barrier $\Delta \Phi(21)$ decreases from $\Delta \Phi_{1}$ to $\Delta \Phi_{2}$.

In contrast, if the total flow rate decreases from $q_{\mathrm{sum}}=q_{\mathrm{sum}}^{(1)}$ to $q_{\mathrm{sum}}=q_{\mathrm{sum}}^{(3)}$, which is close to the threshold flow rate $q_{\mathrm{th}}^{(B)}(19)$ for random traffic breakdown, then the potential barrier $\Delta \Phi$ (21) increases from $\Delta \Phi_{1}$ to $\Delta \Phi_{3}$ (Fig. 4). At the threshold point $q_{\text {sum }}=q_{\mathrm{th}}^{(\mathrm{B})}(19)$, the potential barrier $\Delta \Phi(N)$ reaches the maximum value

$$
\Delta \Phi=\Phi\left(N_{s}\right)-\Phi\left(N_{\mathrm{th}}\right)
$$

where $N_{\mathrm{th}}=N_{1}$ at $q_{\mathrm{sum}}=q_{\mathrm{th}}^{(\mathrm{B})}$. As a result, the mean time delay $T_{\mathrm{FS}}^{(\mathrm{B}, \text { mean })}$ (20) strongly increases as $q_{\text {sum }}$ approaches the threshold point $q_{\mathrm{th}}^{(\mathrm{B})}$. Under the condition

$$
q_{\mathrm{sum}}<q_{\mathrm{th}}^{(\mathrm{B})}
$$

no traffic breakdown at the bottleneck regardless of a random increase in the vehicle number within the cluster is possible at the bottleneck.

If in the vicinity of the critical vehicle number $N_{\mathrm{d}}$ the function $w_{-}(N)(14)$ can be approximated by a parabolic function of $N$, then the following approximate formula can be derived from (20) (Appendix A):

$$
T_{\mathrm{FS}}^{(\mathrm{B}, \text { mean })}=\frac{\sqrt{2} \pi N_{\mathrm{d}}}{q_{\text {determ, FS }}^{(\mathrm{B})}\left(\xi_{\mathrm{d}} \Delta_{\mathrm{c}}\right)^{1 / 2}}\left(\frac{1+\Delta_{\mathrm{c}}^{1 / 2}}{1-\Delta_{\mathrm{c}}^{1 / 2}}\right)^{2 \sqrt{2 / \xi_{\mathrm{d}}} N_{\mathrm{d}}} \exp \left(-\frac{4 \sqrt{2} N_{\mathrm{d}} \Delta_{\mathrm{c}}^{1 / 2}}{\sqrt{\xi_{\mathrm{d}}}}\right),
$$

where

$$
\xi_{\mathrm{d}}=-\left.\left(N^{2} d^{2} \ln w_{-} / d N^{2}\right)\right|_{N=N_{\mathrm{d}}}
$$


is a dimensionless value of the order of 1 ,

$$
\Delta_{\mathrm{c}}=\frac{q_{\mathrm{determ}, \mathrm{FS}}^{(\mathrm{B})}-q_{\mathrm{sum}}}{q_{\text {determ, FS }}^{(\mathrm{B})}}
$$

i.e., $\Delta_{\mathrm{c}}$ is the relative difference between the critical flow rate $q_{\text {determ, FS }}^{(\mathrm{B})}$ for the deterministic $\mathrm{F} \rightarrow \mathrm{S}$ transition and the total flow rate $q_{\text {sum }}(3)$. If in (28) $\Delta_{\mathrm{c}} \ll 1$, then we get

$$
T_{\mathrm{FS}}^{(\mathrm{B}, \text { mean })}=\frac{\sqrt{2} \pi N_{\mathrm{d}}}{q_{\text {determ, FS }}^{(\mathrm{B})}\left(\xi_{\mathrm{d}} \Delta_{\mathrm{c}}\right)^{1 / 2}} \exp \left(\frac{8 N_{\mathrm{d}} \Delta_{\mathrm{c}}^{3 / 2}}{3 \sqrt{2 \xi_{\mathrm{d}}}}\right) .
$$

Respectively, the nucleation rate $G_{\mathrm{FS}}^{(\mathrm{B})}=1 / T_{\mathrm{FS}}^{(\mathrm{B} \text {, mean) }}$ for traffic breakdown at the bottleneck associated with (31) is

$$
G_{\mathrm{FS}}^{(\mathrm{B})}=\frac{q_{\mathrm{determ}, \mathrm{FS}}^{(\mathrm{B})}\left(\xi_{\mathrm{d}} \Delta_{\mathrm{c}}\right)^{1 / 2}}{\sqrt{2} \pi N_{\mathrm{d}}} \exp \left(-\frac{8 N_{\mathrm{d}} \Delta_{\mathrm{c}}^{3 / 2}}{3 \sqrt{2 \xi_{\mathrm{d}}}}\right) .
$$

Note that $q_{\text {determ, FS }}^{(\mathrm{B})}, N_{\mathrm{d}}$, and $\xi_{\mathrm{d}}$ depend $q_{\mathrm{on}}$. Therefore, the mean time delay $T_{\mathrm{FS}}^{(\mathrm{B}, \text { mean })}(31)$ and the nucleation rate $G_{\mathrm{FS}}^{(\mathrm{B})}(32)$ are functions of $q_{\text {sum }}$ and $q_{\mathrm{on}}$.

If the flow rate $q_{\text {on }}$ decreases continuously up to a small enough value (however, we assume that $q_{\mathrm{on}}>0$, i.e., the deterministic cluster still exists at the bottleneck), then the values $\xi_{\mathrm{d}}, N_{\mathrm{d}}$, and $q_{\text {determ, FS }}^{(\mathrm{B})}$ in (30), (32) and, therefore, the nucleation rate $G_{\mathrm{FS}}^{(\mathrm{B})}(32)$ do not decrease proportionally to this decrease in

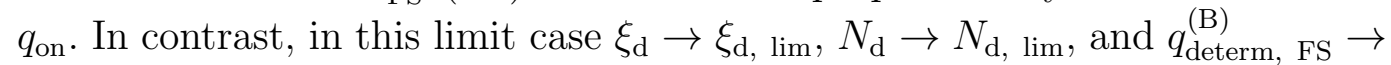
$q_{\text {determ, lim }}^{(\mathrm{B})}$, where $\xi_{\mathrm{d} \text {, lim }}, N_{\mathrm{d}}$, lim, and $q_{\mathrm{determ}}^{(\mathrm{B})} \lim$ are constants. Taking into account that in this case in $\Delta_{\mathrm{c}}(30)$ the flow rate $q_{\text {determ, } \mathrm{FS}}^{(\mathrm{B})} \approx q_{\text {determ, lim }}^{(\mathrm{B})}=$ const, we can see that at small enough values of $q_{\text {on }}$ the nucleation rate for traffic breakdown (32) depends on the total flow rate $q_{\text {sum }}$ only. In other words, in this limit case at a given $q_{\text {sum }}$ within the flow rate range (17) the nucleation rate for traffic breakdown at the bottleneck (32) tends to a finite constant value, which is greater than zero (see Sect. 4.1).

When $q_{\mathrm{on}}=0$, the road can be considered homogeneous one without bottlenecks. Then there is no deterministic perturbation (deterministic cluster) at the bottleneck and, therefore, the nucleation model and results of this article cannot be applied. In three-phase traffic theory, the breakdown phenomenon can also occur in this case. However, at the same conditions, in particlular, the same flow rates downstream of the bottleneck and on a homogeneous road, the nucleation rate for traffic breakdown on the homogeneous road is considerably smaller than at the bottleneck $[33,3]$. This is associated with empirical 
results in which the breakdown phenomenon has also been observed away from bottlenecks, however, this traffic breakdown is much more rare than at an onramp bottleneck [3]. A consideration of a nucleation model of the breakdown phenomenon for a homogeneous road is beyond the scope of this article.

As usual for each first-order phase transition observed in many other systems in natural science [38], the nucleation rate for traffic breakdown (32) is an exponentional function of $\Delta_{c}(30)$. For traffic flow, in accordance with (32) and (30) the exponential growth of the nucleation rate with $\Delta_{c}(30)$ is very sensible to the critical value for the deterministic breakdown phenomenon $q_{\text {determ, FS }}^{(\mathrm{B})}$ This emphasizes the important impact of the deterministic cluster, which occurs at the bottleneck at $q_{\text {on }}>0$, on the nucleation rate for traffic breakdown (32) at a given total flow rate $q_{\text {sum }}$.

\section{Discussion}

4.1 Numerical simulations of general results of nucleation model for traffic breakdown

Let us compare general results of the nucleation model presented in Sect. 3 with the diagram of congested patterns at an on-ramp bottleneck postulated in [30] and found in numerical simulations of microscopic traffic flow models $[31,32]$, as well as with a microscopic theory of the breakdown phenomenon [33]. To reach this goal, we consider an example of the function $w_{-}$ $(14)$

$$
w_{-}(N)=N\left[\frac{a}{1+\left(N / N_{0}\right)^{4}}+b\right]
$$

where $a, b$, and $N_{0}$ are functions of $q_{\text {on }}: a\left(q_{\text {on }}\right)=1.32 q_{0}\left(q_{\mathrm{on}}\right) / N_{0}\left(q_{\mathrm{on}}\right) 1 / \mathrm{h}$, $q_{0}\left(q_{\text {on }}\right)=2700+370\left(1+q_{\text {on }} / 300\right)^{-1}$ vehicles $/ \mathrm{h}, b\left(q_{\text {on }}\right)=33+10\left(1+q_{\text {on }} / 250\right)^{-1}$ $1 / \mathrm{h}, N_{0}\left(q_{\mathrm{on}}\right)=25-6.5\left(1+q_{\mathrm{on}} / 300\right)^{-1}$ vehicles; the unit of $q_{\text {on }}$ is vehicles $/ \mathrm{h}$.

The analytical function (33) allows us to perform a numerical analysis of the mean time delay (20) and the associated nucleation rate (24) for the breakdown phenomenon $(\mathrm{F} \rightarrow \mathrm{S}$ transition). For the analysis of (20) and (24), only branches $N^{\text {(determ) }}$ and $N_{\text {c }}$ (Fig. 3) of the function (33) associated with the deterministic and critical clusters within which $N \leq N_{\mathrm{s}}$ are relevant. This is because the maximum possible value of $N=N_{2}$ in the potential barrier $\Delta \Phi$ (21) that determines the nucleation rate (24) is equal to $N_{\mathrm{s}}$.

However, for a qualitative illustration of a possible synchronized flow state re- 

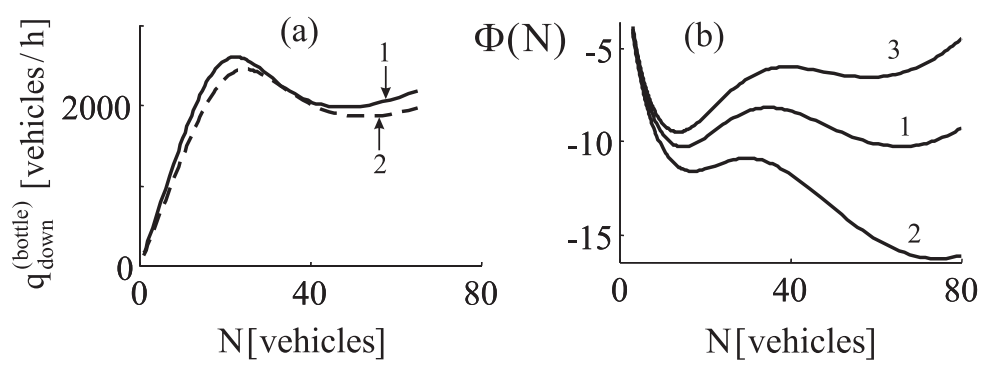

Fig. 5. N-shaped function $q_{\text {down }}^{\text {(bottle) }}(N)(33)$ (a) for $q_{\text {on }}=100$ vehicles $/$ h (curve 1) and $q_{\mathrm{on}}=600$ vehicles $/ \mathrm{h}$ (curve 2 ), and the associated potential $\Phi(22)$ (b) as functions of the vehicle number $N$ for $q_{\text {on }}=100$ vehicles $/$ h and for three different total flow rates $q_{\text {sum }}: 2070$ (curve 3), 2200 (curve 1), 2400 vehicles/h (curve 2).

sulting from an $\mathrm{F} \rightarrow \mathrm{S}$ transition, in (33) the branch for the synchronized flow state is added, in which the detachment rate $w_{-}(N)$ increases with $N^{3}$. This branch corresponds to $N>N_{\mathrm{s}}$. Respectively, this branch of the detachment rate $w_{-}(N)$ has no influence on the analysis of (20) and (24). For this reason, a simple mathematical approximation (33) of the latter branch of $w_{-}(N)$ is chosen, in which the detachment rate $w_{-}(N)$ exhibits formally unlimited growth with $N$. As mentioned, this does not impact on results discussed below. Moreover, as follows from (A.4) (see Appendix) probability of cluster emergence, which size $N$ is large, is negligible.

A numerical study shows that the potential $\Phi$ exhibits qualitatively the same behavior at different total flow rates $q_{\text {sum }}$ (Fig. 5 (b)) as those in Fig. 4 . The potential barrier $\Delta \Phi$ in $(20)$, (24) (Fig. 6 (a)) and the associated critical vehicle number difference $\Delta N_{\mathrm{c}}(18)$ are decreasing functions of the total flow rate $q_{\text {sum }}$; at a given $q_{\text {sum }}$ they can also be decreasing functions of $q_{\text {on }}$ (Fig. 6

3 For more detail explanation of the approximation (33), note that as in the general model (Fig. 3), the function (33) is a N-shape flow-vehicle-number characteristic (Fig. 5 (a)). This N-shape is chosen to satisfy those results of a microscopic threephase traffic theory [3] in which a Z-shaped speed-flow characteristic for an $\mathrm{F} \rightarrow \mathrm{S}$

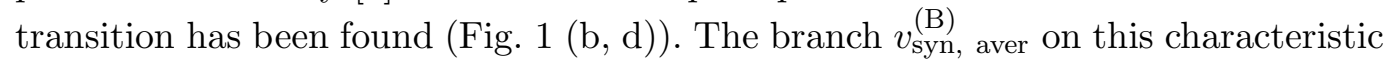
(Fig. $1(\mathrm{~d})$ ) as well as the associated branch $N^{(\mathrm{syn})}$ on the N-shape flow-vehiclenumber characteristic (Fig. 4 (a)) are associated with a synchronized flow state, which results from the $\mathrm{F} \rightarrow \mathrm{S}$ transition. The greater the density, i.e., the vehicle number $N$ within the synchronized flow state, the greater the flow rate $q_{\text {down }}^{\text {(bottle) }}$ (Fig. 1 (c, e)), i.e., the detachment rate $w_{-}(N)$ (14). In real traffic flow, the growth of $w_{-}(N)$ with $N$ has obviously a limit. This limit is related to spontaneous moving jam emergence in synchronized flow of lower speed and greater density (i.e., greater $N)$. In this case, an SP transforms into an GP, which consists of two traffic phases, synchronized flow and wide moving jams [3]. However, these effects are beyond the scope of this article. As mentioned above, the simple mathematical approximation (33) of the branch of $w_{-}(N)$ for synchronized flow states that are associated with $N>N_{\mathrm{s}}$ can be used, because at $N>N_{\mathrm{s}}$ the function $w_{-}(N)$ has no impact on results presented in the article. 

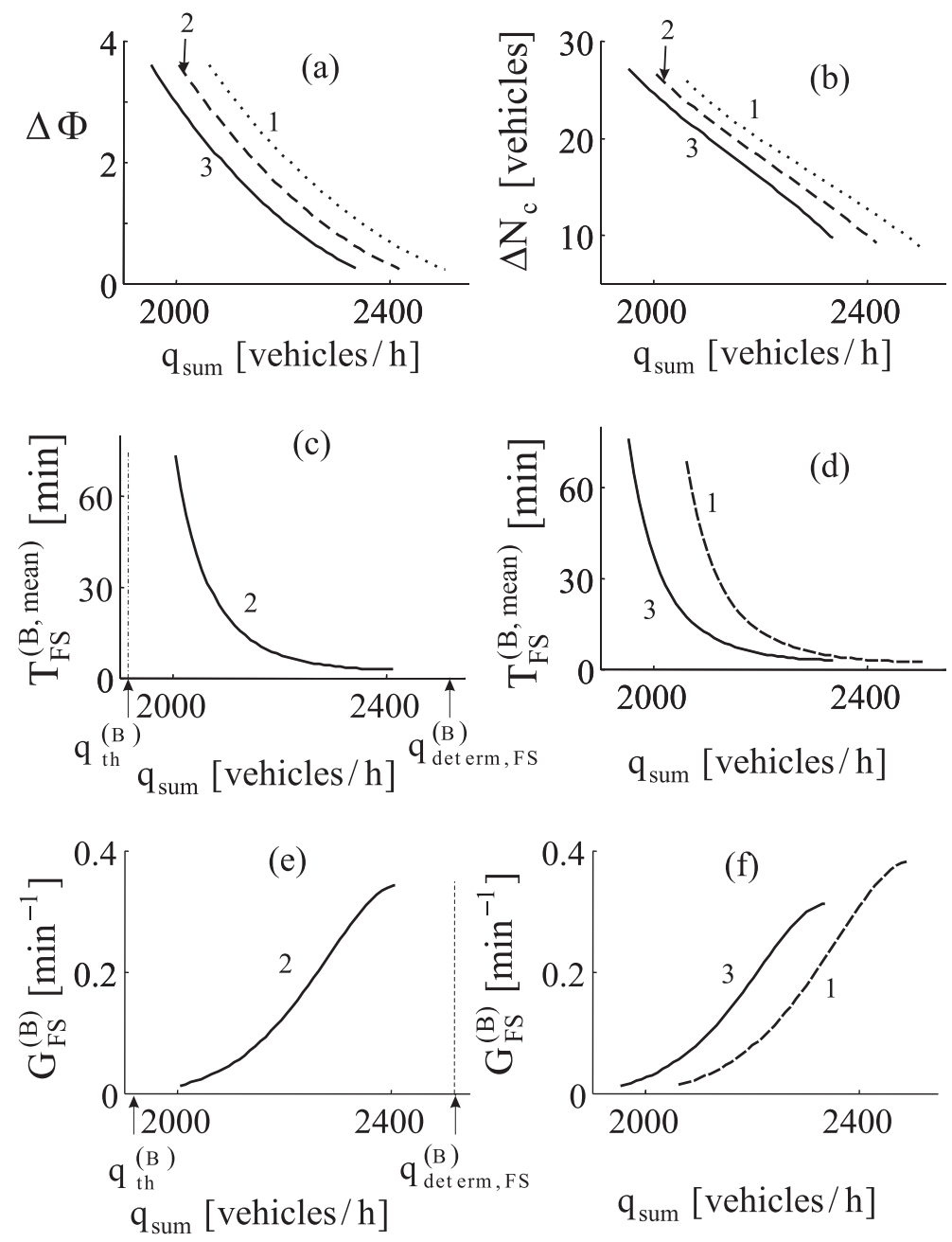

Fig. 6. Potential barrier $\Delta \Phi(21)$ (a), critical vehicle number difference $\Delta N_{\mathrm{c}}$ (18) (b), mean time delay for traffic breakdown $T_{\mathrm{FS}}^{(\mathrm{B}, \text { mean) }}(20)$ (c, d), and nucleation rate for traffic breakdown $G_{\mathrm{FS}}^{(\mathrm{B})}(24)(\mathrm{e}, \mathrm{f})$ as functions of the total flow rate $q_{\text {sum }}$ for three different flow rates $q_{\text {on }}$ : 100 (curves 1), 300 (curves 2), 800 (curves 3) vehicles/h.

(b)). For these reasons, the total flow rate dependences of the mean time delay $T_{\mathrm{FS}}^{(\mathrm{B}, \text { mean })}(20)$ (Fig. $6(\mathrm{c}, \mathrm{d})$ ) and of the associated nucleation rate for traffic breakdown at the bottleneck (Fig. $6(\mathrm{e}, \mathrm{f})$ ) exhibit qualitative features observed in traffic flow at on-ramp bottlenecks $[8,9]$ and found in a microscopic three-phase traffic theory $[32,33]$. This confirms that the breakdown phenomenon at the bottleneck is a first-order $\mathrm{F} \rightarrow \mathrm{S}$ transition [3]. In all these curves, the total flow rate $q_{\text {sum }}$ is smaller than the critical flow rate for the deterministic traffic breakdown $q_{\text {determ, FS }}^{(\mathrm{B})}(4)$. This means that $\Delta_{\mathrm{c}}(30)$ is not equal zero for all results in Fig. 6, i.e., traffic breakdown occurs due to a random density increase within an initial deterministic cluster at the bottleneck. The total flow rate dependences of the nucleation rate for traffic breakdown $G_{\text {FS }}^{(\mathrm{B})}(24)$ calculated at different flow rates $q_{\text {on }}$ exhibit features of three-phase traffic theory in which the breakdown phenomenon can also occur at small 

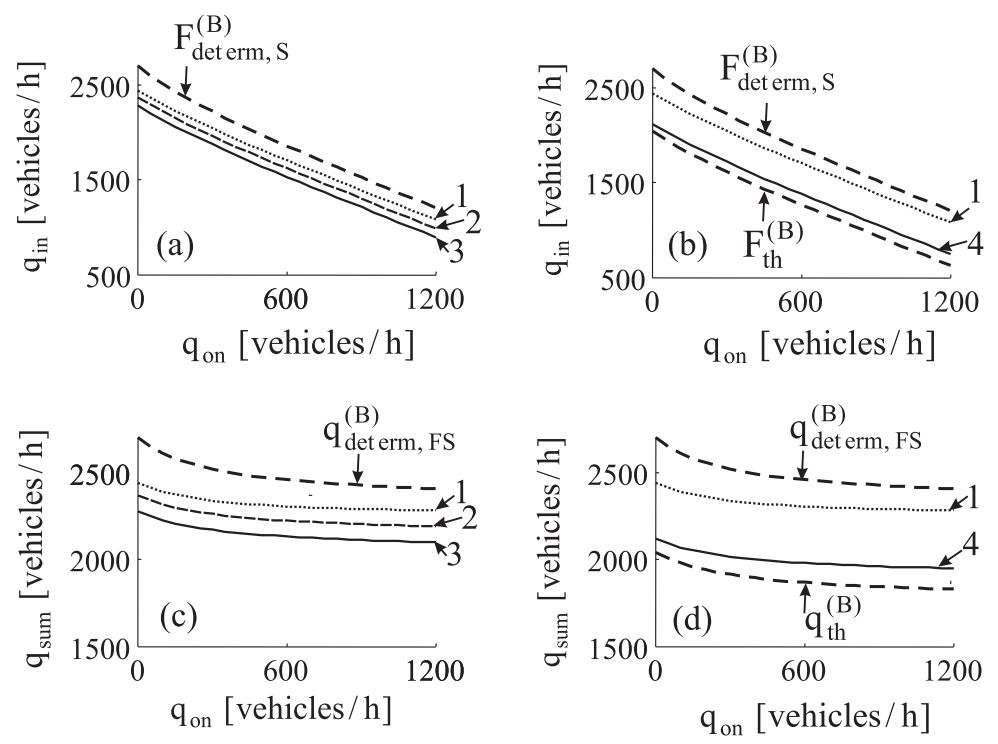

Fig. 7. Characteristics of the nucleation model: $(\mathrm{a}, \mathrm{b})$ - Boundaries of constant values of the nucleation rate $G_{\mathrm{FS}}^{(\mathrm{B})}$ of traffic breakdown (curves 1-4), the critical boundary $F_{\text {determ, S }}^{(\mathrm{B})}$ for deterministic traffic breakdown (curves $F_{\text {determ, S }}^{(\mathrm{B})}$ ), and the threshold boundary $F_{\text {th }}^{(\mathrm{B})}$ (curve $F_{\text {th }}^{(\mathrm{B})}$ ) as functions of the flow rates $q_{\mathrm{on}}$ and $q_{\text {in }}$. (c, d) Dependencies of the flow rate $q_{\mathrm{G}}^{(\mathrm{B})}(35)$ (curves $1-4$ ), the critical flow rate $q_{\text {determ, FS }}^{(\mathrm{B})}$ (4) for deterministic traffic breakdown (curves $q_{\text {determ, FS }}^{(\mathrm{B})}$ ), and the threshold flow rate $q_{\mathrm{th}}^{(B)}(19)$ (curve $q_{\mathrm{th}}^{(B)}$ ) as functions of $q_{\mathrm{on}}$. Curves $1-4$ are related to different given values $\zeta$ for the nucleation rate of traffic breakdown $G_{\mathrm{FS}}^{(\mathrm{B})}$ in $(34): 1 / 3.5$ (curves 1), 0.2 (curves 2), 0.1 (curves 3), 1/60 (curves 4) $\mathrm{min}^{-1}$. The nucleation model cannot be applied for $q_{\text {on }}=0$, therefore, the points in all figures in the vicinity of $q_{\text {on }}=0$ show only the tendency of the boundaries in $(\mathrm{a}, \mathrm{b})$ and the flow rates in (c, d) for the limiting case of small values $q_{\text {on }}$ in which, however, the on-ramp inflow rate $q_{\text {on }}>0$, specifically, it is assumed that the deterministic cluster still exists at the bottleneck.

values $q_{\text {on }}$.

The critical boundary $F_{\mathrm{S}, \zeta}^{(\mathrm{B})}(\mathrm{Fig} .7(\mathrm{a}, \mathrm{b}))$ in the diagram of congested patterns at the bottleneck (flow-flow plane with the coordinates $\left(q_{\text {on }}, q_{\text {in }}\right)$ ) is associated with the cases in which the nucleation rate for traffic breakdown is a given value $\zeta$. Therefore, the boundary $F_{\mathrm{S}, \zeta}^{(\mathrm{B})}$ satisfies the condition

$$
G_{\mathrm{FS}}^{(\mathrm{B})}\left(q_{\mathrm{sum}}, q_{\mathrm{on}}\right)=\zeta, \quad \zeta=\mathrm{const}
$$

i.e., at the boundary $F_{\mathrm{S}, \zeta}^{(\mathrm{B})}$ the flow rate

$$
q_{\mathrm{sum}}=q_{\mathrm{G}}^{(\mathrm{B})}\left(q_{\mathrm{on}}\right)
$$


depends on $q_{\text {on }}$. This boundary is qualitatively similar with the critical boundary $F_{\mathrm{S}}^{(\mathrm{B})}$ in the diagram at which the probability for traffic breakdown for a given time $T_{\mathrm{ob}}$ for observing traffic flow is 1 [3]. In the diagram, there is also the threshold boundary $F_{\mathrm{th}}^{(\mathrm{B})}$ (curve $F_{\mathrm{th}}^{(\mathrm{B})}$ in Fig. $7(\mathrm{~b})$ ) at which the condition (19) is satisfied. The threshold boundary also exhibits the same qualitative features as those found in simulation of phase transitions and spatiotemporal congested patterns in a microscopic three-phase traffic theory [33,3]. In particular, in the limiting case of small values $q_{\text {on }}$ (but $q_{\text {on }}>0$, i.e., it is assumed that the deterministic cluster still exists at the bottleneck) the flow rate $q_{\mathrm{G}}^{(\mathrm{B})}$ reaches the maximum (limit) value $q_{\mathrm{G}}^{(\mathrm{B})}$, lim at a given nucleation rate for traffic breakdown $\zeta(34)$.

The greater the nucleation rate for traffic breakdown, the greater $q_{\mathrm{G} \text {, lim }}^{(\mathrm{B})}$ should be. However, the increase in nucleation rate for traffic breakdown has a limit associated with deterministic traffic breakdown occurrence: When $\zeta$ in (34) increases, the boundary $F_{\mathrm{S}, \zeta}^{(\mathrm{B})}$ for random traffic breakdown tends to the boundary $F_{\text {determ, S }}^{(\mathrm{B})}$ (curves $F_{\text {determ, s }}^{(\mathrm{B})}$ in Fig. $7(\mathrm{a}, \mathrm{b})$ ) for deterministic traffic breakdown in the diagram of congested patterns. At the boundary $F_{\text {determ, S }}^{(\mathrm{B})}$, the deterministic breakdown phenomenon occurs within the deterministic cluster even if no random vehicle number increase within the deterministic cluster appears at the bottleneck. When $\zeta$ in $(34)$ decreases, the boundary $F_{\mathrm{S}, \zeta}^{(\mathrm{B})}$ tends to the threshold boundary $F_{\text {th }}^{(\mathrm{B})}$ (curve $F_{\text {th }}^{(\mathrm{B})}$ in Fig. $7(\mathrm{~b})$ ). In accordance with a microscopic theory [33], in the nucleation model the flow rate $q_{\mathrm{G}}^{(\mathrm{B})}$ (35) (curves 1-4 in Fig. $7(\mathrm{c}, \mathrm{d}))$, the critical flow rate $q_{\text {determ, FS }}^{(\mathrm{B})}$ for deterministic traffic breakdown (curves $q_{\text {determ, Fs }}^{(\mathrm{B})}$ in Fig. $7(\mathrm{c}, \mathrm{d})$ ), as well as the threshold flow rate $q_{\mathrm{th}}^{(B)}(19)$ (curve $q_{\mathrm{th}}^{(B)}$ in Fig. $\left.7(\mathrm{~d})\right)$ can be the smaller, the greater $q_{\text {on }}$ is.

\subsection{Comparison with earlier nucleation models for traffic breakdown}

In $[13,14,15]$ nucleation models for traffic breakdown for a homogeneous circular road have been developed (see Fig. 11 in the review [40]). However, rather than traffic breakdown $(\mathrm{F} \rightarrow \mathrm{S}$ transition), in $[13,14,15]$ a nucleation theory for wide moving jam emergence in an initially homogeneous free flow $(\mathrm{F} \rightarrow \mathrm{J}$ transition) has been derived. Indeed, in final results of this probabilistic theory the vehicle speed within the vehicle cluster is chosen to be zero and a fundamental diagram for traffic flow with the vehicle cluster derived in the probabilistic theory [15] is qualitatively the same (see Fig. 48 in [40]) as those first found in [10] in a macroscopic theory of free flow metastability associated with $\mathrm{F} \rightarrow \mathrm{J}$ transition. This fundamental diagram is confirmed by empirical results associated with wide moving jam propagation (see Fig. 17 in [40]). However, even on a homogeneous road, traffic breakdown is governed by an 
$\mathrm{F} \rightarrow \mathrm{S}$ transition rather than by an $\mathrm{F} \rightarrow \mathrm{J}$ transition [3]. Thus, the nucleation theory of $[13,14,15,40]$ does not describe traffic breakdown on a homogeneous road.

The $\mathrm{F} \rightarrow \mathrm{S}$ transition that can occur away from freeway bottlenecks is a very rare event [3]. This is because a freeway bottleneck introduces a spatial nonhomogeneity in free flow at the road. The average speed within this nonhomogeneity, which is permanently localized in a neighborhood of the bottleneck location, is lower and the vehicle density is greater than on the road away from the bottleneck [3]. This explains why in empirical observations traffic breakdowns are mostly observed at bottlenecks $[7,8,9,3]$.

The first nucleation model for traffic breakdown at an on-ramp bottleneck has been suggested by Kühne, Mahnke et al. [41,40]. In this model (see Chap. 17 in [40]), a hypothesis of three-phase traffic theory about the sequence of the $\mathrm{F} \rightarrow \mathrm{S} \rightarrow \mathrm{J}$ transitions that governs phase transitions at the bottleneck [3] have been taken into account. In addition, in accordance with this theory [3] a random vehicle cluster, whose occurrence can lead to an $\mathrm{F} \rightarrow \mathrm{S}$ transition, is localized at the bottleneck $[41,40]$.

However, in this nucleation model a random vehicle precluster that emerges from fluctuations is necessary. This precluster, which consists of one vehicle $(n=1)$, should occur in an initial hypothetical unperturbed free flow at bottleneck in which no vehicle cluster exists before. The precluster, which can be associated with a random decrease in speed of one of the vehicle in a neighborhood of the bottleneck, foregoes subsequent vehicle cluster evolution towards a critical cluster (critical nuclei) for traffic breakdown $[41,40]$. The attachment rate of precluster formation $w_{+}(0)$ is equal to the flow rate to the on-ramp $q_{\text {on }}[41,40]$ :

$$
w_{+}(0)=q_{\text {on }} \text {. }
$$

The attachment rate (36) of vehicle precluster formation does not depend on the flow rate $q_{\text {in }}$ upstream of the bottleneck. However, in real traffic flow both flow rates $q_{\text {on }}$ and $q_{\text {in }}$ exhibit random fluctuations. The formula (36) should be associated with the basic model assumption that at $q_{\mathrm{on}}=0$ no vehicle cluster can randomly appear, specifically no traffic breakdown is possible $[41,40]$. Apparently the assumption (36) leads to the nucleation rate for an $\mathrm{F} \rightarrow \mathrm{S}$ transition at the bottleneck that is proportional to the flow rate to the on-ramp $[41,40]$.

Whereas for a homogeneous road the model assumption for the necessity of precluster formation $[13,14,15]$ is physically justified, this is not the case for the nucleation model at the bottleneck introduced in $[41,40]$. To explain this, note that in contrast with the model of a homogeneous road, at $q_{\text {on }}>0$ and $q_{\text {in }}>0$ 
an initial state of free flow at the bottleneck is non-homogeneous regardless of fluctuations. This means that even no fluctuations would occur in free flow at the bottleneck, nevertheless free flow is non-homogeneous in a neighborhood of the bottleneck [3]. This is because two different flows permanent merge within the merging region of the on-ramp - the flow with the rate $q_{\text {on }}$ and the flow with the rate $q_{\text {in }}$. Vehicles merging from the on-ramp onto the main road force vehicles on the main road to slow down. In turn, these slower moving vehicles on the main road force vehicles merging from the on-ramp onto the main road to decrease the speed too. Thus, the speed is lower and the density is greater at the bottleneck, i.e., a local cluster appears regardless of fluctuations. Thus, a permanent and motionless (deterministic) vehicle cluster in which speed is lower and the density is greater than away from bottleneck exists already on the road, even if there were no fluctuations in traffic flow. For this reason, the formula $(36)[41,40]$ that assumes no vehicle cluster existence without random fluctuations in free flow at the bottleneck is in serious conflict with empirical results and results a microscopic three-phase traffic theory [3].

Moreover, the master equation of this model [41,40] searches the probability for a random vehicle cluster with $n$ vehicles. In this master equation, a vehicle cluster exists $(n>0)$ only then, if the precluster has appeared. When there were no fluctuations at the bottleneck at all, then a vehicle cluster cannot appear $(n=0)$ and no traffic breakdown is possible in the model. In contrast, in three-phase traffic theory deterministic traffic breakdown is possible regardless of fluctuations. This deterministic traffic breakdown occurs when the size of the deterministic cluster exceeds some critical value. This is the consequence of the non-homogeneity in free flow at the bottleneck mentioned above.

Thus, in contrast with the above basic assumptions of Ref. [41,40], a nucleation model that can be adequate with empirical results should search probability $p$ for random vehicle cluster evolution in which the cluster size $N$ randomly changes due to fluctuations in a neighborhood of the deterministic cluster (Fig. 2). The size of this deterministic cluster $N^{(\text {determ) }}$ does not depend on fluctuations in traffic flow. Random fluctuation either increases the speed within the cluster or decreases it. Consequently, the density and the cluster size $N$ either decreases or increases. In the latter case, traffic breakdown occurs at a smaller flow rate $q_{\text {sum }}=q_{\text {in }}+q_{\text {on }}$ than the critical flow rate $q_{\text {sum }}=q_{\text {determ FS }}^{(\mathrm{B})}$ associated with the deterministic traffic breakdown that occurs without any fluctuations at the bottleneck.

These fundamental differences in the nucleation model of Ref. [41,40] and the model presented in this article can explain different results of these models. In $[41,40]$, even if the on-ramp inflow rate $q_{\text {on }}$ is high, in an initial steady state of traffic flow there is no deterministic cluster at the bottleneck. As a result, there is no deterministic breakdown phenomenon of three-phase traffic theory in this model. Probably for this reason, in the model $[41,40]$ the nucleation 
rate for the breakdown phenomenon (generation rate of traffic breakdown critical nuclei) is proportional to the on-ramp inflow rate $q_{\text {on }}$. As a result, if $q_{\text {on }}$ decreases below a small enough value (but $q_{\text {on }}>0$ ) and the total flow rate $q_{\text {sum }}$ increases (through an increase in $q_{\text {in }}$ ), a reasonable given nucleation rate for traffic breakdown at the bottleneck (the nucleation rate should be greater than $\approx 1 / 20 \mathrm{~min}^{-1}$, in accordance with empirical observations $[8,9]$ ) cannot be reached. This is true in the nucleation model of $[41,40]$ even if the total flow rate is equal to a critical value associated with the critical nuclei for traffic breakdown consisting of one vehicle only (in $[41,40]$ this critical value is denoted by $q_{\mathrm{c} 2}$ ).

In contrast, in our model the nucleation rate (generation rate of critical nuclei) for the breakdown phenomenon is not proportional to $q_{\text {on }}$ and, therefore, as mentioned in Sect. 3, for the limiting case of small values $q_{\text {on }}$ (however, we assume that $q_{\text {on }}>0$, specifically, the deterministic cluster still exists at the bottleneck) this generation rate of critical nuclei depends on the total flow rate $q_{\text {sum }}$ only, i.e., this generation rate does not depend on $q_{\text {on }}$. At a given $q_{\text {sum }}$, an increase in $q_{\text {on }}$ can influence only on such characteristics of traffic breakdown as the critical flow rate $q_{\text {determ, Fs }}^{(\mathrm{B})}$ and the threshold flow rate $q_{\mathrm{th}}^{(\mathrm{B})}$, as well as on congested traffic states at the bottleneck that result from the breakdown phenomenon.

When $q_{\text {sum }}$ increases, the nucleation rate of traffic breakdown increases in the both models. However, in our model the nucleation rate cannot exceed the nucleation rate for traffic breakdown associated with the deterministic breakdown phenomenon. In contrast with assumptions of the nucleation model of Ref. [41,40], in our nucleation model the deterministic traffic breakdown occurs even without any random vehicle number increase within the initial steady state of free flow at the bottleneck. This is because if $q_{\text {on }}>0$, then in our model there is a deterministic vehicle cluster localized at the bottleneck, which exists permanent at the bottleneck due to the on-ramp inflow. In our model, random traffic breakdown nucleation can occurs through a random increase in vehicle number within this deterministic cluster. The mentioned qualitative differences in the nucleation model of Ref. [41,40] and our nucleation model are also responsible for different dependences of the generation rate of traffic breakdown on the total flow rate in these nucleation models.

\section{A Derivation of nucleation rate}

In order to derive formula (20), we use a general formula for the mean time delay $T$ of escaping from the potential well for the master equation (6) [38]: 


$$
T=\sum_{n=N_{1}}^{N_{3}}\left[\left(w_{+} p_{\mathrm{s}}(n)\right)^{-1} \sum_{k=0}^{n} p_{\mathrm{s}}(k)\right],
$$

where $p_{\mathrm{s}}(N)$ is a steady solution of $(6),(7)$ :

$$
p_{\mathrm{s}}(N)=p_{\mathrm{s}}(0) \prod_{n=1}^{N} \frac{w_{+}}{w_{-}(n)} \quad \text { at } N>0 .
$$

When $N_{\mathrm{i}} \gg 1, i=1,2$ (more rigorous conditions are given below), the distribution $p_{\mathrm{s}}(N)$ has a sharp maximum at $N=N_{1}$, and the function $p_{\mathrm{s}}^{-1}(n)$ in (A.1) has a sharp maximum at $n=N_{2}$. Then the formula (A.1) can be written as follows [38]:

$$
T=\left(w_{+}\right)^{-1} \sum_{n=0}^{N_{2}} p_{\mathrm{s}}(n) \sum_{n=N_{1}}^{N_{3}} p_{\mathrm{s}}^{-1}(n) .
$$

Formula (A.2) can be written as

$$
p_{\mathrm{s}}(N)=p_{\mathrm{s}}(0) \exp [-\Phi(N)] \quad \text { at } N \geq 0,
$$

where the potential $\Phi(N)$ is given by (22). Substituting (A.4) into (A.3), we can find the exponentially large factor in (A.3) explicitly

$$
T=\left(w_{+}\right)^{-1} c_{1} c_{2} \exp \left[\Phi\left(N_{2}\right)-\Phi\left(N_{1}\right)\right]
$$

where

$$
\begin{aligned}
& c_{1}=\sum_{n=0}^{N_{2}} \exp \left[-\Delta \Phi^{(1)}(n)\right], c_{2}=\sum_{n=N_{1}}^{N_{3}} \exp \left[\Delta \Phi^{(2)}(n)\right], \\
& \Delta \Phi^{(i)}(N)=\Phi(N)-\Phi\left(N_{i}\right), \quad i=1,2 .
\end{aligned}
$$

The factors $c_{1}, c_{2}$ can be estimated using the parabolic approximation of potential $\Phi(N)$ near the extremum points $N=N_{1}, N_{2}$ [39]. For instance, to find the factor $c_{1}$, we introduce a new variable $y=N / N_{1}$ and approximate the sums in (22), (A.6) by integrals:

$$
\begin{array}{r}
\Delta \Phi^{(1)}(N) \approx \phi^{(1)}(y)=N_{1} \int_{1}^{y} \ln \frac{w_{-}\left(N_{1} z\right)}{w_{+}} d z, \\
c_{1} \approx N_{1} \int_{0}^{N_{2} / N_{1}} \exp \left[-\phi^{(1)}(y)\right] d y .
\end{array}
$$


Using the series expansion

$$
\phi^{(1)}(y)=N_{1} \eta_{1}(y-1)^{2} / 2+O\left((y-1)^{3}\right)
$$

near the point $y=1$, where $\eta_{1}=d \ln w_{-} /\left.d \ln N\right|_{N=N_{1}}$, we find $c_{1}=\sqrt{2 \pi N_{1} / \eta_{1}}$. Similarly, $c_{2}=\sqrt{2 \pi N_{2} / \eta_{2}}$, where $\eta_{2}=-d \ln w_{-} /\left.d \ln N\right|_{N=N_{2}}$. The substitution of $c_{1}$ and $c_{2}$ into (A.5) yields the formula (20).

The parabolic approximation used for estimation of factor $c_{1}$ holds only when we can neglect in integral (A.9) third-order terms in the potential expansion (A.10) [39]. The same is true for calculation of $c_{2}$. The conditions of the parabolic approximation are

$$
N_{i} \eta_{i}^{3} \gtrsim \xi_{i}^{2}, \quad i=1,2
$$

where $\xi_{i}=N_{i}^{2} d^{2} \ln w_{-} /\left.d N^{2}\right|_{N=N_{i}}, i=1,2$.

To derive the formula (28), we approximate the value $\Delta \Phi$ in (20) by integral

$$
\Delta \Phi=N_{1} \int_{1}^{N_{2} / N_{1}} \ln \frac{w_{-}\left(N_{1} y\right)}{w_{+}} d y
$$

Under approximation of the function $w_{-}(N)$ in (A.12) near the maximum point $N=N_{\mathrm{d}}$ by parabola, we get:

$$
w_{-}(N)=q_{\text {determ, FS }}^{(\mathrm{B})}\left[1-\xi_{\mathrm{d}} \frac{\left(N-N_{\mathrm{d}}\right)^{2}}{2 N_{\mathrm{d}}^{2}}\right]
$$

where the formula $q_{\text {determ, FS }}^{(\mathrm{B})}=w_{-}\left(N_{\mathrm{d}}\right)$ is taken into account. The roots $N=$ $N_{1}$ and $N=N_{2}$ of equation $q_{\text {sum }}=w_{-}(N)$ given by formulae (14), (16) are

$$
N_{1,2}=N_{d} \mp \Delta N_{\mathrm{c}} / 2,
$$

where the critical value $\Delta N_{\mathrm{c}}(18)$ is

$$
\Delta N_{\mathrm{c}}=2 \sqrt{2} \xi_{\mathrm{d}}^{-1 / 2} N_{d} \Delta_{\mathrm{c}}^{1 / 2}
$$

Substituting (A.13)-(A.15) into (A.12), and calculating the derivatives $w_{-}^{\prime}\left(N_{i}\right)$ in $(23): w_{-}^{\prime}\left(N_{i}\right) \approx \pm q_{\text {determ, FS }}^{(\mathrm{B})} \xi_{\mathrm{d}} \Delta N_{\mathrm{c}} /\left(2 N_{\mathrm{d}}^{2}\right), i=1,2$, we get $(28)$. 
Under the condition that $q_{\text {sum }}$ is close to the critical point $q_{\text {determ, FS }}^{(\mathrm{B})}$, i.e., when

$$
\Delta_{\mathrm{c}} \ll 1
$$

from (28), we get the approximate formula (31). The latter is applicable under the condition (A.16) only if the conditions (A.11) are still satisfied. Using the formula for derivatives $w_{-}^{\prime}\left(N_{i}\right), i=1,2$ and that $N_{i}, i=1,2$ is close to $N_{\mathrm{d}}$, we can estimate the values $\xi_{i} \approx \xi_{\mathrm{d}}$ and $\eta_{i} \approx \xi_{\mathrm{d}} \Delta N_{\mathrm{c}} / N_{\mathrm{d}}, i=1,2$ in (A.11). Thus, the condition (A.11) reads $\xi_{\mathrm{d}} \Delta N_{\mathrm{c}}^{3} / N_{\mathrm{d}}^{2} \gtrsim 1$. Taking into account formula (A.15) for $\Delta N_{\mathrm{c}}$, the condition (A.11) can be written in terms of $\Delta_{\mathrm{c}}$ :

$$
N_{\mathrm{d}} \Delta_{\mathrm{c}}^{3 / 2} \xi_{\mathrm{d}}^{-1 / 2} \gtrsim 1
$$

This inequality together with (A.16) determine the range of $\Delta_{\mathrm{c}}$ in which the approximate formula (31) for the mean time delay $T_{\mathrm{FS}}^{(\mathrm{B}, \text { mean) }}$ of an $\mathrm{F} \rightarrow \mathrm{S}$ transition at the bottleneck is valid.

\section{References}

[1] F.L. Hall, V.F. Hurdle, J.H. Banks, Transportation Research Record 1365, 12 18 (1992).

[2] J.H. Banks, Transportation Research Record 1802, 225-232 (2002).

[3] B.S. Kerner. The Physics of Traffic (Springer, Berlin, New York 2004).

[4] J.-B. Lesort (editor). Transportation and Traffic Theory, Proceedings of the 13th International Symposium on Transportation and Traffic Theory (Elsevier Science Ltd, Oxford 1996).

[5] A. Ceder (editor). Transportation and Traffic Theory, Proceedings of the 14th International Symposium on Transportation and Traffic Theory (Elsevier Science Ltd, Oxford 1999).

[6] M.A.P. Taylor (editor). Transportation and Traffic Theory in the 21st Century, Proceedings of the 15th International Symposium on Transportation and Traffic Theory (Elsevier Science Ltd, Amsterdam 2002).

[7] L. Elefteriadou, R.P. Roess, W.R. McShane, Transportation Research Record 1484, 80-89 (1995).

[8] B.N. Persaud, S. Yagar, R. Brownlee: Transportation Research Record 1634, 64-69 (1998).

[9] M. Lorenz, L. Elefteriadou, Transportation Research Circular E-C018, 84-95 (2000). 
[10] B.S. Kerner, P. Konhäuser, Phys. Rev. E 50, 54-83 (1994).

[11] M. Bando, K. Hasebe, A. Nakayama, A. Shibata, Y. Sugiyama, Phys. Rev. E 51, 1035-1042 (1995).

[12] M. Schreckenberg, A. Schadschneider, K. Nagel, N. Ito, Phys. Rev. E 51, 2939 (1995).

[13] R. Mahnke, N. Pieret, Phys. Rev. E 56, 2666 (1997).

[14] R. Mahnke, J. Kaupužs, Phys. Rev. E 59, 117 (1999).

[15] R. Kühne, R. Mahnke, I. Lubashevsky, J. Kaupužs, Phys. Rev. E 66066125 (2002).

[16] D. Chowdhury, L. Santen, A. Schadschneider. Physics Reports 329, 199 (2000).

[17] D. Helbing. Rev. Mod. Phys. 73, 1067-1141 (2001).

[18] T. Nagatani. Rep. Prog. Phys. 65, 1331-1386 (2002).

[19] K. Nagel, P. Wagner, R. Woesler. Operation Res. 51, 681-716 (2003).

[20] D.E. Wolf, M. Schreckenberg, A. Bachem (editors). Traffic and Granular Flow, Proceedings of the International Workshop on Traffic and Granular Flow, October 1995 (World Scientific, Singapore 1995).

[21] M. Schreckenberg, D.E. Wolf (editors). Traffic and Granular Flow' 97, Proceedings of the International Workshop on Traffic and Granular Flow, October 1997 (Springer, Singapore 1998)

[22] D. Helbing, H.J. Herrmann, M. Schreckenberg, D.E. Wolf (editors). Traffic and Granular Flow' 99, Proceedings of the International Workshop on Traffic and Granular Flow, October 1999, (Springer, Heidelberg 2000).

[23] M. Fukui, Y. Sugiyama, M. Schreckenberg, D.E. Wolf (editors). Traffic and Granular Flow' 01, Proceedings of the International Workshop on Traffic and Granular Flow, October 2001, (Springer, Heidelberg 2003).

[24] S.P. Hoogendoorn, P.H.L. Bovy, M. Schreckenberg, D.E. Wolf (editors). Traffic and Granular Flow' 03, Proceedings of the International Workshop on Traffic and Granular Flow, October 2003, (Springer, Heidelberg 2005) (in press).

[25] B.S. Kerner, P. Konhäuser, M. Schilke, Phys. Rev. E 51, 6243-6246 (1995).

[26] D. Helbing, A. Hennecke, M. Treiber: Phys. Rev. Lett. 82, 4360 (1999).

[27] M. Treiber, A. Hennecke, D. Helbing, Phys. Rev. E 62, 1805-1824 (2000).

[28] H.Y. Lee, H.-W. Lee, D. Kim: Phys. Rev. E 59, 5101 (1999).

[29] B. S. Kerner, Phys. Rev. Lett. 81, 3797 (1998).

[30] B. S. Kerner, Phys. Rev. E 65, 046138 (2002).

[31] B.S. Kerner, S.L. Klenov: J. Phys. A: Math. Gen. 35, L31 (2002). 
[32] B.S. Kerner, S.L. Klenov, D.E. Wolf, J. Phys. A: Math. Gen. 35 9971-10013 (2002).

[33] B.S. Kerner, S.L. Klenov, Phys. Rev. E 68036130 (2003).

[34] B.S. Kerner, S.L. Klenov, J. Phys. A: Math. Gen. 37 8753-8788 (2004).

[35] L.C. Davis, Phys. Rev. E 69016108 (2004).

[36] H.K. Lee, R. Barlović, M. Schreckenberg, D. Kim, Phys. Rev. Let. 92, 238702 (2004).

[37] R. Jiang, Q.S. Wu, J. Phys. A: Math. Gen. 37, 8197-8213 (2004).

[38] C. W. Gardiner, Handbook of Stochastic Methods for Physics, Chemistry, and the Natural Sciences (Springer, Berlin, 1994).

[39] N. G. van Kampen, Stochastic Processes in Physics and Chemistry (NorthHolland Physics Publishing, 1984).

[40] R. Mahnke, J. Kaupužs, I. Lubashevsky, Phys. Rep. 408, 1-130 (2005).

[41] R. Kühne, I. Lubashevsky, R. Mahnke, J. Kaupužs, cond-mat/0405163 (2004).

[42] B.S. Kerner, Transportation Research Record 1710, 136 (2000).

[43] B.S. Kerner, V.V. Osipov, Autosolitons: A New Approach to Problems of SelfOrganization and Turbulence (Kluwer, Dordrecht, Boston, London 1994).

[44] A.S. Mikhailov, Foundations of Synergetics Vol. I (Springer, Berlin 1994), 2nd ed. 